\title{
ON A CONJECTURE OF LAN-SHENG-ZUO ON SEMISTABLE HIGGS BUNDLES: RANK 3 CASE
}

\author{
LINGGUANG LI
}

\begin{abstract}
Let $X$ be a smooth projective curve of genus $g$ over an algebraically closed field $k$ of characteristic $p>2$. We prove that any rank 3 nilpotent semistable Higgs bundle $(E, \theta)$ on $X$ is a strongly semistable Higgs bundle. This gives a partially affirmative answer to a conjecture of Lan-Sheng-Zuo $\left[8{ }^{*}\right.$. In addition, we prove a tensor product theorem for strongly semistable Higgs bundles with $p$ satisfying some bounds (Theorem 4.3). From this we reprove a tensor theorem for semistable Higgs bundles on the condition that the LanSheng-Zuo conjecture holds (Corollary 4.4).
\end{abstract}

\section{INTRODUCTION}

N. Hitchin [5, K. Corlette [3] 4] and C. Simpson [13, 15] have established a correspondence between semistable Higgs bundles and representations of the fundamental groups on arbitrary dimensional complex projective manifolds. In order to establish an analogous correspondence in positive characteristic, Lan-Sheng-Zuo [8] introduced intermediate notions strongly semistable Higgs bundles and quasiperiodic Higgs bundles between semistable Higgs bundles and representations of algebraic fundamental groups, where strongly semistable Higgs bundles is a generalization of the notion of strongly semistable vector bundles in the sense of LangeStuhler [11. They constructed a functor from the category of strongly semistable Higgs bundles with trivial Chern classes to the category of crystalline representations of fundamental groups, and showed that any rank 2 nilpotent semistable Higgs bundle is a strongly semistable Higgs bundle (cf. [8]). In addition, they made the following conjecture.

Conjecture. Any nilpotent semistable Higgs bundle of exponent less than the characteristic of base field is a strongly semistable Higgs bundle.

In this paper, we show that any rank 3 nilpotent semistable Higgs bundle is a strongly semistable Higgs bundle. This gives an affirmative answer to the conjecture of Lan-Sheng-Zuo [8] in the rank 3 case.

Theorem 1.1 (Theorem 3.3). Let $k$ be an algebraically closed field of characteristic $p>2, X$ a smooth projective curve over $k$. Let $(E, \theta)$ be a rank 3 nilpotent semistable Higgs bundle on $X$. Then $(E, \theta)$ is a strongly semistable Higgs bundle.

\footnotetext{
${ }^{*}$ Very recently, A. Langer [1] and independently Lan-Sheng-Yang-Zuo [9] has proven the conjecture for ranks less than or equal to $p$ case.

This work is supported by the SFB/TR 45 'Periods, Moduli Spaces and Arithmetic of Algebraic Varieties' of the DFG, and partially supported by the National Natural Science Foundation (No. 11271275).
} 
In fact, the theorem above is also true in higher dimension since the proof is valid in the higher dimension case. With the results of Lan-Sheng-Zuo [8, the theorem above implies that we can construct crystalline representations of fundamental groups associated to rank 3 nilpotent semistable Higgs bundles with trivial Chern classes.

By the equivalence of strongly semistable Higgs bundles and quasi-periodic Higgs bundles over an algebraic closure of finite fields, we can prove the following tensor product theorem for strongly semistable Higgs bundles with trivial Chern classes.

Theorem 1.2 (Theorem 4.3). Let $k$ be the algebraic closure of finite fields of characteristic $p>0$, and $X$ a smooth projective curve over $k$. Let $\left(E_{1}, \theta_{1}\right)$ and $\left(E_{2}, \theta_{2}\right)$ be strongly semistable Higgs bundles on $X$ with degree 0 . Suppose that $\operatorname{rk}\left(E_{1}\right)+\operatorname{rk}\left(E_{2}\right) \leq p+1$. Then the tensor product $\left(E_{1} \otimes E_{2}, \theta_{1} \otimes 1+1 \otimes \theta_{2}\right)$ is also a strongly semistable Higgs bundle.

Professor Kang Zuo explained to me that the idea in the proof is a flavor of a characteristic $p$ analog to the complex number field case. Over the complex number field, S. K. Donaldson, N. Hitchin and C. Simpson showed that a Higgs bundle is poly-stable if and only if it carries a Yang-Mills-Higgs metric (for semistable Higgs bundles one has the so-called approximated Yang-Mills-Higgs metric). If the degree of this vector bundle is zero, then one shows that the tensor product of Yang-MillsHiggs metrics is again a Yang-Mills-Higgs metric. In this way, one proves the above theorem over the complex number field. For the characteristic $p$ case, the solution of Lan-Sheng-Zuo conjecture just means that any semistable Higgs bundle in characteristic $p$ of degree zero carries a characteristic $p$ Yang-Mills-Higgs metric. The above theorem can be interpreted as the tensor product of Yang-Mills-Higgs metrics in characteristic $p$ is again a Yang-Mills-Higgs metric in characteristic $p$.

If the Lan-Sheng-Zuo conjecture is true, one can reprove a tensor theorem for semistable Higgs bundles (cf. [2, Theorem 8.16]).

Corollary 1.3 (Corollary 4.4). Suppose that Lan-Sheng-Zuo conjecture is true, i.e. any nilpotent semistable Higgs bundle of exponent less than the characteristic of base field is strongly Higgs semistable. If $\left(E_{1}, \theta_{1}\right)$ and $\left(E_{2}, \theta_{2}\right)$ are nilpotent semistable Higgs bundles on $X$ with degree 0 and $\operatorname{rk}\left(E_{1}\right)+\operatorname{rk}\left(E_{2}\right) \leq p+1$, then the tensor product $\left(E_{1} \otimes E_{2}, \theta_{1} \otimes 1+1 \otimes \theta_{2}\right)$ is also Higgs semistable.

\section{Preliminary}

Let $X / \mathbb{C}$ be a smooth projective scheme over the complex number field, $(E, \nabla)$ a de Rham bundle on $X$ which satisfies suitable conditions. C. Simpson [13, constructed a Higgs bundle $\left(E^{\prime}, \theta\right)$ associated to $(E, \nabla)$. Later A. Ogus and V. Vologodsky 12 established a correspondence between category of de Rham bundles and category of Higgs bundles in positive characteristic using the theory of Azumaya algebra and certain universal algebra. Lan-Sheng-Zuo [7] gave a explicit construction of inverse Cartier transform for nilpotent Higgs bundles of exponent $\leq p-1$ which is equivalent to the correspondence in [12.

Now, let's first recall the definitions of strongly semistable Higgs bundle and quasi-periodic Higgs bundle. Let $k$ be an algebraically closed field of characteristic $p>0, X$ a smooth projective curve of genus $g$ over $k$, and $F: X \rightarrow X$ the absolute 
Frobenius morphism. A Higgs-de Rham sequence over $X$ is a sequence of form

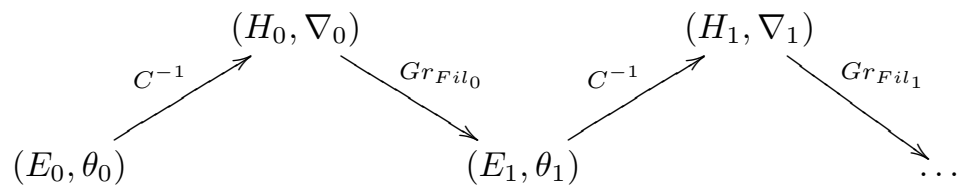

where $C^{-1}$ is the inverse Cartier transform, $F i l_{i}$ is a decreasing filtration on $H_{i}$ satisfies Griffiths transversality, $\left(H_{i}, \nabla_{i}\right)=C^{-1}\left(E_{i}, \theta_{i}\right)$ is a de Rham bundle and $\left(E_{i}, \theta_{i}\right)=\operatorname{Gr}_{F i l_{i-1}}\left(H_{i-1}, \nabla_{i-1}\right)$ is a Higgs bundle on $X$.

Definition 2.1. A Higgs bundle $(E, \theta)$ is called strongly Higgs semistable if it appears in the leading term of a Higgs-de Rham sequence whose Higgs terms $\left(E_{i}, \theta_{i}\right) \mathrm{s}$ are all Higgs semistable.

Remark 2.2. The definition of strongly Higgs semistable has been modified in the new version ([10, Definition 2.2]) of the Definition 2.1 in [8]. But this does not affect our proof below.

Definition 2.3. A Higgs bundle $(E, \theta)$ is called quasi-periodic if it appears in the leading term of a quasi-periodic Higgs-de Rham sequence, i.e., it becomes periodic after a nonnegative integer.

Lemma 2.4. Let $k$ be an algebraically closed field, $X$ a smooth projective curve over $k$. Let $(H, \nabla)$ be a $\nabla$-semistable flat bundle on $X, H^{\prime}$ a subsheaf of $H$ with $\mu\left(H^{\prime}\right)>\mu(H)$. Then the homomorphism $H^{\prime} \rightarrow\left(H / H^{\prime}\right) \otimes_{\mathfrak{O}_{X}} \Omega_{X}^{1}$ is non-trivial.

Proof. Suppose that $H^{\prime}$ is invariant under $\nabla$, then $\mu\left(H^{\prime}\right) \leq \mu(H)$ by the $\nabla$ semistability of $(H, \nabla)$. A contradiction.

Remark 2.5. Suppose that the characteristic of $k$ is $p>0$. Let $(E, \theta)$ be a nilpotent semistable Higgs bundle of exponent $\leq p-1$ on $X$ and $(H, \nabla):=C^{-1}(E, \theta)$. Then $(H, \nabla)$ is $\nabla$-semistable by [12, Theorem 2.8].

\section{Rank 3 semistable Higgs Bundles are Strongly Higgs semistable}

In this section, unless otherwise explicitly declared, we have the following notations and assumptions:

Let $k$ be an algebraically closed field of characteristic $p>0, X$ a smooth projective curve of genus $g \geq 0$ over $k$, and $(E, \theta)$ a rank 3 nilpotent semistable Higgs bundle of exponent $\leq p-1$ on $X$. Denote $(H, \nabla):=C^{-1}(E, \theta)$, where $C^{-1}$ is the inverse Cartier transform constructed in $[12$ and $[7$. We would like to construct a Hodge filtration $\left\{F i l_{H}^{i}\right\}$ of $H$ with respect to the connection $\nabla$ such that the grading $\operatorname{Gr}_{F i l_{H}^{*}}(H, \nabla)$ is a semistable Higgs bundle.

Suppose that $H$ is a semistable bundle, we can choose the trivial filtration $F i l_{t r i}$ on $H$, then there is nothing need to prove. We assume that $H$ is not a semistable bundle. Let $S \subseteq H$ (resp. $H \rightarrow Q$ ) be the subbundle (resp. quotient bundle) of $H$ with maximal (resp. minimal) slope in the Harder-Narasimhan filtration of $H$.

Proposition 3.1. Let $Q^{\prime}:=H / S, \theta: S \hookrightarrow H \stackrel{\nabla}{\rightarrow} H \otimes_{\mathscr{O}_{X}} \Omega_{X}^{1} \rightarrow Q^{\prime} \otimes_{\mathscr{O}_{X}} \Omega_{X}^{1}$ the natural homomorphism, and $\widetilde{S}$ the saturation of $\operatorname{Im}(\theta) \otimes_{\mathscr{O}_{X}} T_{X}$ in $Q^{\prime}$. Suppose that $\operatorname{rk}(S)=1$ and $\mu_{\max }\left(Q^{\prime}\right) \leq \mu(H)$. Then 
(I) If $\mu\left(Q^{\prime} / \widetilde{S}\right) \leq \mu(H)$, then $\left(S \oplus \widetilde{S} \oplus\left(Q^{\prime} / \widetilde{S}\right), \bigoplus_{i=1}^{2} \theta^{i}\right)$ is a semistable Higgs bundle;

(II) If $\mu\left(Q^{\prime} / \widetilde{S}\right) \geq \mu(H)$, then $\left(S \oplus Q^{\prime}, \theta\right)$ is a semistable Higgs bundle.

Proof. For the case $\mu(H)=0$, the assumption of this proposition can be explained in the following diagram (The general case is similar to the slope zero case after rotate this diagram along the origin).

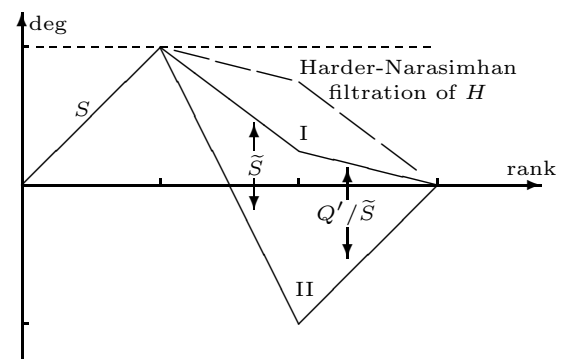

(I) Let $F$ be a nonzero Higgs subsheaf of $\left(S \oplus \widetilde{S} \oplus\left(Q^{\prime} / \widetilde{S}\right), \bigoplus_{i=1}^{2} \theta^{i}\right)$.

Case I.i: The composition $F \hookrightarrow S \oplus \widetilde{S} \oplus\left(Q^{\prime} / \widetilde{S}\right) \rightarrow Q^{\prime} / \widetilde{S}$ is trivial, i.e. $F \subset S \oplus \widetilde{S}$.

Suppose that $\operatorname{rk}(F)=1$. Then the composition $F \hookrightarrow S \oplus \widetilde{S} \rightarrow \widetilde{S}$ is not trivial. Otherwise, we have $F \subset S$, this contradicts to the fact that $\theta^{1}: S \hookrightarrow \widetilde{S} \otimes_{\mathscr{O}_{X}} \Omega_{X}^{1}$ is injective by Lemma 2.4. Thus $\mu(F) \leq \mu(\widetilde{S}) \leq \mu_{\max }\left(Q^{\prime}\right) \leq \mu(H)$.

Suppose that $\operatorname{rk}(F)=2$. Then we can get that $\theta^{2}: \widetilde{S} \hookrightarrow\left(Q^{\prime} / \widetilde{S}\right) \otimes_{\mathscr{O}_{X}} \Omega_{X}^{1}$ is trivial and $\mu\left(Q^{\prime} / \widetilde{S}\right)=\mu(H)$. Thus $\mu(F) \leq \mu(S \oplus \widetilde{S})=\mu(H)$.

Case I.ii: The composition $F \hookrightarrow S \oplus \widetilde{S} \oplus\left(Q^{\prime} / \widetilde{S}\right) \rightarrow Q^{\prime} / \widetilde{S}$ is not trivial.

Suppose that $\operatorname{rk}(F)=1$. Then the injection $F \hookrightarrow S \oplus \widetilde{S} \oplus\left(Q^{\prime} / \widetilde{S}\right) \rightarrow Q^{\prime} / \widetilde{S}$ implies that $\mu(F) \leq \mu(Q / \widetilde{S}) \leq \mu(H)$.

Suppose that $\operatorname{rk}(F)=2$. Then $\operatorname{rk}(F \cap(S \oplus \widetilde{S}))=1$ and the composition $F \cap(S \oplus \widetilde{S}) \hookrightarrow S \oplus \widetilde{S} \rightarrow \widetilde{S}$ is injective, since $\theta^{1}: S \hookrightarrow \widetilde{S} \otimes_{\mathscr{O}_{X}} \Omega_{X}^{1}$ is injective by Lemma 2.4. Consider the commutative diagram

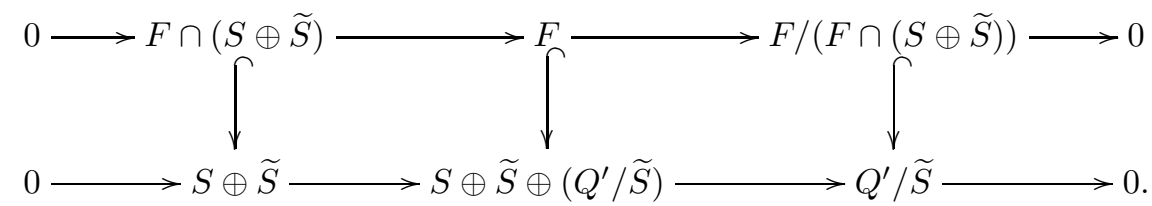

Then we have

$$
\begin{aligned}
\mu(F) & =\frac{1}{2}[\mu(F \cap(S \oplus \widetilde{S}))+\mu(F /(F \cap(S \oplus \widetilde{S})))] \\
& \leq \frac{1}{2}\left[\mu(\widetilde{S})+\mu\left(Q^{\prime} / \widetilde{S}\right)\right] \\
& <\mu(H) .
\end{aligned}
$$

Hence $\left(S \oplus \widetilde{S} \oplus\left(Q^{\prime} / \widetilde{S}\right), \bigoplus_{i=1}^{2} \theta^{i}\right)$ is a semistable Higgs bundle.

$(I I)$ Let $F$ be a nonzero Higgs subsheaf of $\left(S \oplus Q^{\prime}, \theta\right)$. Then the composition $F \hookrightarrow S \oplus Q^{\prime} \rightarrow Q^{\prime}$ is not trivial, since $\theta: S \hookrightarrow Q^{\prime} \otimes_{\mathscr{O}_{X}} \Omega_{X}^{1}$ is not trivial by Lemma 2.4 . 
Suppose that $\operatorname{rk}(F)=1$. Then the injection $F \hookrightarrow S \oplus Q^{\prime} \rightarrow Q^{\prime}$ implies that $\mu(F) \leq \mu_{\max }\left(Q^{\prime}\right) \leq \mu(H)$.

Suppose that $\operatorname{rk}(F)=2$. If the composition $F \hookrightarrow S \oplus Q^{\prime} \rightarrow Q^{\prime}$ is injective, then $\mu(F) \leq \mu\left(Q^{\prime}\right)<\mu(H)$. If the composition $F \hookrightarrow S \oplus Q^{\prime} \rightarrow Q^{\prime}$ is not injective, then $\operatorname{rk}(F \cap S)=\operatorname{rk}\left(F \cap Q^{\prime}\right)=1$. Consider the following commutative diagram

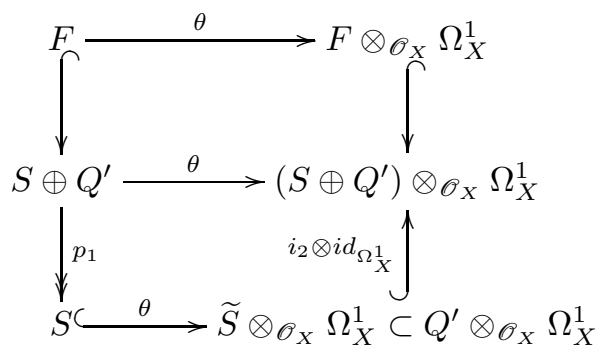

where $p_{1}: S \oplus Q^{\prime} \rightarrow S$ is the natural projection and $i_{2}: Q^{\prime} \hookrightarrow S \oplus Q^{\prime}$ is the natural injection. Thus $F \cap \widetilde{S} \neq 0$. Therefore, the saturations of $F \cap \widetilde{S}$ and $F \cap Q^{\prime}$ in $Q^{\prime}$ are the same, since $F \cap \widetilde{S} \subseteq F \cap Q^{\prime}$ with $\operatorname{rk}(F \cap \widetilde{S})=\operatorname{rk}\left(F \cap Q^{\prime}\right)=1$. It follows that $F \cap Q^{\prime} \subseteq \widetilde{S}$, since $F \cap \widetilde{S} \subseteq \widetilde{S}$ and $\widetilde{S}$ is a subbundle of $Q^{\prime}$. Consider the commutative diagram

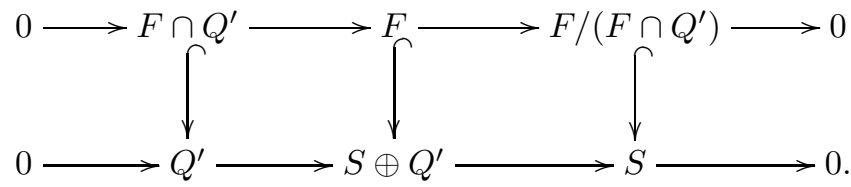

Then we have

$$
\begin{aligned}
\mu(F) & =\frac{1}{2}\left[\mu\left(F \cap Q^{\prime}\right)+\mu\left(F /\left(F \cap Q^{\prime}\right)\right)\right] \\
& \leq \frac{1}{2}[\mu(\widetilde{S})+\mu(S)] \\
& =\frac{1}{2}\left[3 \mu(H)-\mu\left(Q^{\prime} / \widetilde{S}\right)\right] \\
& \leq \mu(H)
\end{aligned}
$$

Hence $\left(S \oplus Q^{\prime}, \theta\right)$ is a semistable Higgs bundle.

Proposition 3.2. Let $S^{\prime}:=\operatorname{ker}(H \rightarrow Q), \theta: S^{\prime} \hookrightarrow H \stackrel{\nabla}{\rightarrow} H \otimes_{\mathscr{O}_{X}} \Omega_{X}^{1} \rightarrow Q \otimes_{\mathscr{O}_{X}} \Omega_{X}^{1}$ the natural homomorphism, and $K:=\operatorname{ker}\left(\theta: S^{\prime} \rightarrow Q \otimes_{\mathscr{O}_{X}} \Omega_{X}^{1}\right)$. Suppose that $\operatorname{rk}(Q)=1$ and $\mu_{\min }\left(S^{\prime}\right) \geq \mu(H)$. Then

(I) If $\mu(K) \leq \mu(H)$, then $\left(S^{\prime} \oplus Q, \theta\right)$ is a semistable Higgs bundle;

(II) If $\mu(K) \geq \mu(H)$, then $\left(K \oplus\left(S^{\prime} / K\right) \oplus Q, \bigoplus_{i=1}^{2} \theta^{i}\right)$ is a semistable Higgs bundle.

Proof. For the case $\mu(H)=0$, the assumption of this proposition can be explained in the following diagram (The general case is similar to the slope zero case after rotate this diagram along the origin). 


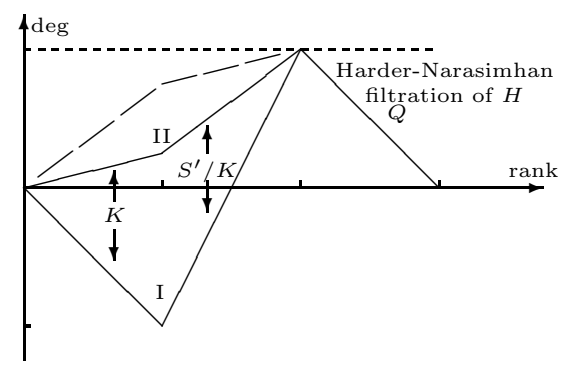

$(I)$ Let $F$ be a nonzero Higgs subsheaf of $\left(S^{\prime} \oplus Q, \theta\right)$.

Suppose that $\operatorname{rk}(F)=1$. If the composition $F \hookrightarrow S^{\prime} \oplus Q \rightarrow Q$ is not trivial, then $\mu(F) \leq \mu(Q)<\mu(H)$. If the composition $F \hookrightarrow S^{\prime} \oplus Q \rightarrow Q$ is trivial, then $F \subseteq K$ since $K=\operatorname{ker}\left(S^{\prime} \rightarrow Q \otimes_{\mathscr{O}_{X}} \Omega_{X}^{1}\right)$. Thus $\mu(F) \leq \mu(K) \leq \mu(H)$.

Suppose that $\operatorname{rk}(F)=2$. Then the composition $F \hookrightarrow S^{\prime} \oplus Q \rightarrow Q$ is not trivial. Consider the commutative diagram

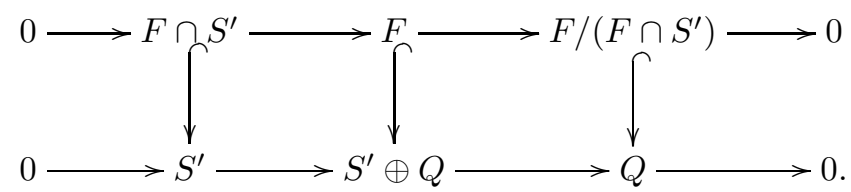

Therefore, we have

$$
\begin{aligned}
\mu(F) & =\frac{1}{2}\left[\mu\left(F \cap S^{\prime}\right)+\mu\left(F /\left(F \cap S^{\prime}\right)\right)\right] \leq \frac{1}{2}\left[\mu_{\max }\left(S^{\prime}\right)+\mu(Q)\right] \\
& =\frac{1}{2}\left[3 \mu(H)-\mu_{\min }\left(S^{\prime}\right)\right] \\
& \leq \mu(H) .
\end{aligned}
$$

Hence $\left(S^{\prime} \oplus Q, \theta\right)$ is a semistable Higgs bundle.

(II) Let $F$ be a nonzero Higgs subsheaf of $\left(K \oplus\left(S^{\prime} / K\right) \oplus Q, \bigoplus_{i=1}^{2} \theta^{i}\right)$.

Suppose that $\operatorname{rk}(F)=1$. If the composition $F \hookrightarrow K \oplus\left(S^{\prime} / K\right) \oplus Q \rightarrow Q$ is not trivial, then $\mu(F) \leq \mu(Q)<\mu(H)$. If the composition $F \hookrightarrow K \oplus\left(S^{\prime} / K\right) \oplus Q \rightarrow Q$ is trivial, then $F \subseteq K \subset K \oplus\left(S^{\prime} / K\right)$ since $\left(S^{\prime} / K\right) \rightarrow Q \otimes_{\mathscr{O}_{X}} \Omega_{X}^{1}$ is injective by Lemma 2.4. In this case, we must have $\mu(K)=\mu(H)$. Otherwise, if $\mu(K)>\mu(H)$, then $K \rightarrow\left(S^{\prime} / K\right) \otimes_{\mathscr{O}_{X}} \Omega_{X}^{1}$ is injective by Lemma 2.4 This controdicts to $F \subseteq K$. Thus $\mu(F) \leq \mu(K)=\mu(H)$.

Suppose that $\operatorname{rk}(F)=2$. Then the composition $F \hookrightarrow K \oplus\left(S^{\prime} / K\right) \oplus Q \rightarrow Q$ is not trivial, since $\theta^{2}:\left(S^{\prime} / K\right) \hookrightarrow Q \otimes_{\mathscr{O}_{X}} \Omega_{X}^{1}$ is injective. Moreover, we have

$$
\mu\left(F \cap\left(K \oplus\left(S^{\prime} / K\right)\right)\right) \leq \mu\left(S^{\prime} / K\right) .
$$

In fact, if $\mu(K)>\mu(H)$, then the homomorphism $\theta^{1}: K \hookrightarrow\left(S^{\prime} / K\right) \otimes_{\mathscr{O}_{X}} \Omega_{X}^{1}$ is injective by Lemma 2.4. In this case, the composition $F \cap\left(K \oplus\left(S^{\prime} / K\right)\right) \hookrightarrow$ $K \oplus\left(S^{\prime} / K\right) \rightarrow S^{\prime} / K$ is not trivial, then $\mu\left(F \cap\left(K \oplus\left(S^{\prime} / K\right)\right)\right) \leq \mu\left(S^{\prime} / K\right)$. If $\mu(K)=\mu(H)$, then

$$
\mu\left(F \cap\left(K \oplus\left(S^{\prime} / K\right)\right)\right) \leq \mu_{\max }\left(K \oplus\left(S^{\prime} / K\right)\right)=\mu\left(S^{\prime} / K\right) .
$$




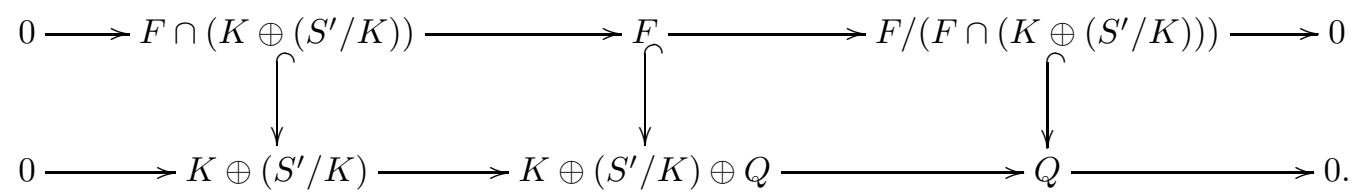

Therefore, we have

$$
\begin{aligned}
\mu(F) & =\frac{1}{2}\left[\mu\left(F \cap\left(K \oplus\left(S^{\prime} / K\right)\right)\right)+\mu\left(F /\left(F \cap\left(K \oplus\left(S^{\prime} / K\right)\right)\right)\right)\right] \\
& \leq \frac{1}{2}\left[\mu\left(S^{\prime} / K\right)+\mu(Q)\right] \\
& =\frac{1}{2}[3 \mu(H)-\mu(K)] \\
& \leq \mu(H)
\end{aligned}
$$

Hence $\left(K \oplus\left(S^{\prime} / K\right) \oplus Q, \bigoplus_{i=1}^{2} \theta^{i}\right)$ is a semistable Higgs bundle.

Combine Proposition 3.1 with Proposition 3.2, we can get the following theorem which is the main result of this section.

Theorem 3.3. Let $k$ be an algebraically closed field of characteristic $p>2, X a$ smooth projective curve over $k$. Let $(E, \theta)$ be a rank 3 nilpotent semistable Higgs bundle on $X$ and $(H, \nabla):=C^{-1}(E, \theta)$. Then there is a Hodge filtration $\left\{F i l_{H}^{*}\right\}$ of $(H, \nabla)$ such that the grading $G_{F i l_{H}^{*}}(H, \nabla)$ is a semistable Higgs bundle. In particular, $(E, \theta)$ is a strongly semistable bundle.

Proof. Assume that $H$ is semistable, we can choose the trivial filtration of $(H, \nabla)$. Otherwise, it is easy to check that $H$ must satisfy one of the following cases

(i) $\operatorname{rk}(S)=1$ and $\mu_{\max }(H / S) \leq \mu(H)$;

(ii) $\operatorname{rk}(Q)=1$ and $\mu_{\min }(\operatorname{ker}(H \rightarrow Q)) \geq \mu(H)$;

where $S \subseteq H$ (resp. $H \rightarrow Q$ ) is the subbundle (resp. quotient bundle) with maximal (resp. minimal) slope in the Harder-Narasimhan filtration of $H$.

In the case $(i)$, we use the notation as Proposition 3.1. If $\mu\left(Q^{\prime} / \widetilde{S}\right) \leq \mu(H)$, we choose the Hodge filtration

$$
0 \subsetneq S \subsetneq \operatorname{ker}\left(H \rightarrow Q^{\prime} \rightarrow\left(Q^{\prime} / \widetilde{S}\right)\right) \subsetneq H .
$$

If $\mu\left(Q^{\prime} / \widetilde{S}\right) \geq \mu(H)$, we choose the Hodge filtration

$$
0 \subsetneq S \subsetneq H \text {. }
$$

Then their gradings are semistable Higgs bundles by Proposition 3.1

In the case (ii), we use the notation as Proposition 3.2. If $\mu(K) \leq \mu(H)$, we choose the Hodge filtration

$$
0 \subsetneq S^{\prime} \subsetneq H
$$

If $\mu(K) \geq \mu(H)$, we choose the Hodge filtration

$$
0 \subsetneq K \subsetneq S^{\prime} \subsetneq H \text {. }
$$

Then their gradings are semistable Higgs bundles by Proposition 3.2

Since the grading $\operatorname{Gr}_{F i l_{H}^{*}}(H, \nabla)$ is a system of Hodge bundle of rank 3, it is a nilpotent semistable Higgs bundle of exponent $\leq 2$. Then we can construct 
inductively a Higgs-de Rham sequence with leading term $(E, \theta)$ such that every Higgs bundle term is Higgs semistable. Hence $(E, \theta)$ is a strongly semistable bundle.

\section{Tensor Product of Strongly semistable Higgs Bundles}

In characteristic 0 case, the tensor product of two semistable vector bundles is still semistable by Kobayashi-Hitchin correspondence. The Kobayashi-Hitchin correspondence in the setting of Higgs bundles has been generalized in a number of ways starting with the far reaching one by C. Simpson [13] and 14. Thus the Higgs semi-stability of tensor product of two semistable Higgs bundles follows from the Higgs bundles version of the Kobayashi-Hitchin correspondence.

In the characteristic $p>0$ case, tensor product of two semistable vector bundles need not be semistable. However, S. Ilangovan, V. B. Mehta, A. J. Parameswaran [6] showed that if $E_{1}$ and $E_{2}$ are semistable vector bundles with $\operatorname{rk}\left(E_{1}\right)+\operatorname{rk}\left(E_{2}\right) \leq$ $p+1$, then $E_{1} \otimes E_{2}$ is still a semistable vector bundle. Later, V. Balaji, A. J. Parameswaran [2] generalized the theorem of Ilangovan-Mehta-Parameswaran to the Higgs bundles case, and proved the following tensor product theorem for semistable Higgs bundles.

Theorem 4.1. 2, Theorem 8.16] Let $k$ be an algebraically closed field of characteristic $p>0, X$ a smooth projective curve over $k$. Let $\left(E_{1}, \theta_{1}\right)$ and $\left(E_{2}, \theta_{2}\right)$ be semistable Higgs bundles on $X$ with $\operatorname{det}\left(E_{i}\right) \cong \mathscr{O}_{X}, i=1,2$. Suppose that $\operatorname{rk}\left(E_{1}\right)+\operatorname{rk}\left(E_{2}\right) \leq p+1$. Then the tensor product $\left(E_{1} \otimes E_{2}, \theta_{1} \otimes 1+1 \otimes \theta_{2}\right)$ is also a semistable Higgs bundle.

The approach of V. Balaji, A. J. Parameswaran [2 is very complicated. In this section, we will give another more simple approach to prove a suitable modification of tensor product theorem for strongly semistable Higgs bundles.

In this section, unless otherwise explicitly declared, $k$ is an algebraic closure of finite fields of characteristic $p>0$, and $X$ a smooth projective curve over $k$.

Lemma 4.2. [8, Theorem 2.5] A Higgs bundle $(E, \theta)$ with trivial Chern classes is quasi-periodic if and only if $(E, \theta)$ is strongly Higgs semistable.

One can easily induce the following tensor theorem for strongly semistable bundle by the equivalence of strongly semistable Higgs bundles and quasi-periodic Higgs bundles.

Theorem 4.3. Let $k$ be the algebraic closure of finite fields of characteristic $p>0$, and $X$ a smooth projective curve over $k$. Let $\left(E_{1}, \theta_{1}\right)$ and $\left(E_{2}, \theta_{2}\right)$ be strongly semistable Higgs bundles on $X$ with degree 0 . Suppose that $\operatorname{rk}\left(E_{1}\right)+\operatorname{rk}\left(E_{2}\right) \leq p+1$. Then the tensor product $\left(E_{1} \otimes E_{2}, \theta_{1} \otimes 1+1 \otimes \theta_{2}\right)$ is also a strongly semistable Higgs bundle.

Proof. Since the exponent of a nilpotent Higgs bundle less than its rank. Therefore, $\operatorname{rk}\left(E_{1}\right)+\operatorname{rk}\left(E_{2}\right) \leq p+1$ implies that $\left(E_{1}, \theta_{1}\right) \otimes\left(E_{2}, \theta_{2}\right)$ and $\left(E_{i}, \theta_{i}\right)(i=1,2)$ are nilpotent Higgs bundles of exponent $\leq p-1$. By Lemma 4.2. we have quasiperiodic Higgs-de Rham sequences $\left\{\left(E_{j}^{(i)}, \theta_{j}^{(i)}\right),\left(H_{j}^{(i)}, \nabla_{j}^{(i)}, F i l_{j}^{(i)}\right)\right\}_{j \in \mathbb{Z}_{\geq 0}}$ such that $\left(E_{0}^{(i)}, \theta_{0}^{(i)}\right) \cong\left(E_{i}, \theta_{i}\right), i=1,2$. Since inverse Cartier transform $C^{-1}$ preserve tensor structures, we have

$C^{-1}\left(\left(E_{0}^{(1)}, \theta_{0}^{(1)}\right) \otimes\left(E_{0}^{(2)}, \theta_{0}^{(2)}\right)\right) \cong C^{-1}\left(E_{0}^{(1)}, \theta_{0}^{(1)}\right) \otimes C^{-1}\left(E_{0}^{(2)}, \theta_{0}^{(2)}\right)=\left(H_{0}^{(1)}, \nabla_{0}^{(1)}\right) \otimes\left(H_{0}^{(2)}, \nabla_{0}^{(2)}\right)$. 
Then there is a Hodge filtration $F i l_{0}$ on $\left(H_{0}^{(1)}, \nabla_{0}^{(1)}\right) \otimes\left(H_{0}^{(2)}, \nabla_{0}^{(2)}\right)$ such that

$$
G r_{F i l_{0}}\left(\left(H_{0}^{(1)}, \nabla_{0}^{(1)}\right) \otimes\left(H_{0}^{(2)}, \nabla_{0}^{(2)}\right)\right) \cong G r_{F i l_{0}^{(1)}}\left(H_{0}^{(1)}, \nabla_{0}^{(1)}\right) \otimes G r_{F i l_{0}^{(2)}}\left(H_{0}^{(2)}, \nabla_{0}^{(2)}\right) .
$$

Thus we have $G r \circ C^{-1}\left(\left(E_{0}^{(1)}, \theta_{0}^{(1)}\right) \otimes\left(E_{0}^{(2)}, \theta_{0}^{(2)}\right)\right) \cong\left(E_{1}^{(1)}, \theta_{1}^{(1)}\right) \otimes\left(E_{1}^{(2)}, \theta_{1}^{(2)}\right)$. Hence we can construct inductively a Higgs-de Rham sequence with Higgs bundles terms $\left\{\left(E_{j}^{(1)}, \theta_{j}^{(1)}\right) \otimes\left(E_{j}^{(2)}, \theta_{j}^{(2)}\right)\right\}_{j \in \mathbb{Z}_{\geq 0}}$. As $\left\{\left(E_{j}^{(i)}, \theta_{j}^{(i)}\right),\left(H_{j}^{(i)}, \nabla_{j}^{(i)}, F i l_{j}^{(i)}\right)\right\}_{j \in \mathbb{Z} \geq 0}(i=1,2)$ are quasi-periodic Higgs-de Rham sequences, then $\left\{\left(E_{j}^{(1)}, \theta_{j}^{(1)}\right) \otimes\left(E_{j}^{(2)}, \theta_{j}^{(2)}\right)\right\}_{j \in \mathbb{Z}_{\geq 0}}$ is also a quasi-periodic Higgs bundles sequence. Hence

$$
\left(E_{1}, \theta_{1}\right) \otimes\left(E_{2}, \theta_{2}\right)=\left(E_{1} \otimes E_{2}, \theta_{1} \otimes 1+1 \otimes \theta_{2}\right)
$$

is a strongly semistable Higgs bundles by Lemma 4.2 .

As a consequence from the tensor product theorem for strongly semistable Higgs bundles, one can reprove the tensor product theorem for semistable Higgs bundles if the Lan-Sheng-Zuo conjecture is true.

Corollary 4.4. Suppose that Lan-Sheng-Zuo conjecture is true, i.e. any nilpotent semistable Higgs bundle is strongly Higgs semistable. If $\left(E_{1}, \theta_{1}\right)$ and $\left(E_{2}, \theta_{2}\right)$ are nilpotent semistable Higgs bundles on $X$ with degree 0 and $\operatorname{rk}\left(E_{1}\right)+\operatorname{rk}\left(E_{2}\right) \leq p+1$, then the tensor product $\left(E_{1} \otimes E_{2}, \theta_{1} \otimes 1+1 \otimes \theta_{2}\right)$ is also Higgs semistable.

Acknowledgments: I did the first part of this work during my stay at Johannes Gutenberg Universität Mainz, Germany, as a visiting fellow in 2012. I would like to express my hearty thanks to Professor Kang Zuo, who introduced this subject to me and told me the idea how to prove the Theorem 4.3. This paper would not be possible without his help and discussions. Thanks are also due to both Professor Mao Sheng and Gui-Tang Lan for many helps and effective discussions. I also wish to thank the hospitality of Institut für Mathematik, Johannes Gutenberg Universität Mainz, Germany.

\section{REFERENCES}

[1] A. Langer, Semistable modules over Lie algebroids in positive characteristic, arXiv: 1311.2794, (2013).

[2] V. Balaji, A. J. Parameswaran, Tensor product theorem for Hitchin pairsan algebraic approach, Ann. Inst. Fourier. 61(6) (2011), 2361-2403.

[3] K. Corlette, Flat G-bundles with canonical metrics, J. Diff. Geom. 28 (1988), 361-382.

[4] K. Corlette,Nonabelian Hodge theory. In Differential geometry: geometry in mathematical physics and related topics (Los Angeles, CA, 1990), 125-144, volume 54 of Proc. Sympos. Pure Math., Amer. Math. Soc., Providence, RI, 1993.

[5] N. Hitchin, The self-duality equations on a Riemann surface, Proc. London Math. Soc. 55 (1987), 59-126.

[6] S. Ilangovan, V. B. Mehta, A. J. Parameswaran, Semistability and semisimplicity in representations of low height in positive characteristic, in A tribute to C. S. Seshadri (Chennai, 2002), 271-282, Trends Math., Birkhauser, Basel, 2003.

[7] G.-T. Lan, M. Sheng, K. Zuo, An inverse Cartier transform via exponential in positive characteristic, arXiv:1205.6599 (2012).

[8] G.-T. Lan, M. Sheng, K. Zuo, Semistable Higgs bundles and representations of algebraic fundamental groups: positive characteristic case, arXiv: 1210.8280, (2012).

[9] G.-T. Lan, M. Sheng, Y.-H. Yang, K. Zuo, Semistable Higgs bundles of small ranks are strongly Higgs semistable, arXiv: 1311.2405, (2013).

[10] G.-T. Lan, M. Sheng, K. Zuo, Semistable Higgs bundles, periodic Higgs bundles and representations of algebraic fundamental groups, arXiv: 1311.6424, (2013). 
[11] H. Lange, U. Stuhler, Vektorbündel auf Kurven und Darstellungen der algebraischen Fundamentalgruppe. Math. Z. 156 (1977), 73-83.

[12] A. Ogus, V. Vologodsky, Nonabelian Hodge theory in characteristic p, Publ. Math. Inst. Hautes'etudes Sci. 106 (2007), 1-138.

[13] C. Simpson, Higgs bundles and local systems. Inst. Hautes Études Sci. Publ. Math. 75 (1992), 5-95.

[14] C. Simpson, Moduli of representations of the fundamental group of a smooth projective variety I. Publ. Math. IHES. 79 (1994), 47-129.

[15] C. Simpson, The Hodge filtration on nonabelian cohomology. In Algebraic Geometry-Santa Cruz 1995, 217-281, Proc. Sympos. Pure Math., 62, Part 2, Amer. Math. Soc., Providence, RI, 1997.

Department of Mathematics, Tongui University, Shanghai, P. R. China, School of Mathematical Sciences, Fudan University, Shanghai, P. R. China

E-mail address: LG.Lee@amss.ac.cn 